\title{
Osteopontin/Secreted Phosphoprotein-1 Behaves as a Molecular Brake Regulating the Brain Translocator Protein-Dependent Neuroinflammatory Response to Chronic Viral Infection
}

\section{Farina J Mahmud}

Johns Hopkins University School of Medicine

Yong Du

Johns Hopkins University School of Medicine

Elizabeth Greif

Johns Hopkins University School of Medicine

Thomas Boucher

Johns Hopkins University School of Medicine

Robert F Dannals

Johns Hopkins University School of Medicine

\section{William B Mathews}

Johns Hopkins University School of Medicine

Martin G Pomper

Johns Hopkins University School of Medicine

Polina Sysa-Shah

Johns Hopkins University School of Medicine

Kelly A Metcalf Pate

Johns Hopkins University School of Medicine

Claire Lyons

Johns Hopkins University School of Medicine

\section{Bess Carlson}

Johns Hopkins University School of Medicine

Maria Chacona

Johns Hopkins University School of Medicine

Amanda M Brown ( $\square$ abrown76@jh.edu )

Johns Hopkins University https://orcid.org/0000-0002-4576-9636

Research 
Keywords: neuroimaging, neuroinflammation, microglial activation, DPA-713, Purkinje cells, human immunodeficiency virus type-1

Posted Date: May 28th, 2020

DOI: https://doi.org/10.21203/rs.3.rs-29745/v1

License: (c) (1) This work is licensed under a Creative Commons Attribution 4.0 International License. Read Full License 


\section{Abstract \\ Background}

Osteopontin (OPN) as a secreted signaling protein, is dramatically induced in response to cellular injury and neurodegeneration. Microglial inflammatory responses in the brain are tightly associated with the neuropathologic hallmarks of neurodegenerative disease, but understanding of the molecular mechanisms remains in several contexts, poorly understood.

\section{Methods}

Positron emission tomography (PET) neuroimaging using radioligands to detect increased expression of the translocator protein (TSPO) receptor in the brain, is a non-invasive tool used to track neuroinflammation in living mammals.

\section{Results}

In humanized, chronically HIV-infected mice in which OPN expression was knocked down with functional aptamers, uptake of TSPO radioligand, DPA-713 was markedly upregulated in the hippocampus, cortex, olfactory bulb, cerebellum and significantly increased in other key brain regions analyzed compared to controls. $\mathrm{TSPO}^{+}$microglia were detected by immunolabeling of post-mortem brain tissue, thus validating the neuroimaging findings. Unexpectedly, two types of neurons also selectively stained positively for TSPO. The reactive cells were the specialized neurons of the cerebellum, Purkinje cells, and a subset of tyrosine hydroxylase positive neurons of the substantia nigra. Two-way ANOVA of immunoreactivity revealed that a well-validated marker of microglial activation in brain tissue, ionized calcium-binding adaptor molecule-1 (Iba-1) was significantly increased, in an interaction that depended on HIV replication. Interestingly, similar analyses of TSPO immunoreactivity showed a significant interaction with OPN expression.

\section{Conclusions}

Collectively, these findings using a model of chronic HIV-infection revealed for the first time two key findings: 1) two different pathways of neuroinflammation are activated, and 2) osteopontin acts as a molecular brake regulating in the brain, the inflammatory response to HIV infection.

\section{Background}

Anti-retroviral therapy which suppresses HIV replication to levels that impair its ability to be transmitted has saved and afforded the opportunity of those in the developed world to live a normal lifespan (1-3). However, in the absence of a cure and therapy that selectively blocks HIV transcription, current treatment 
does not stop all immune activation and chronic inflammation. Systemic inflammation is now recognized as a comorbid condition that contributes by differing pathways, to the pathogenesis of many chronic disorders (2). Currently, $30-50 \%$ of HIV-infected persons experience at varying severity neuropsychiatric and cognitive impairment that significantly lowers quality of life $(4,5)$ with mounting evidence suggesting that sex may play a role in differential vulnerability (6). Deficits in learning, memory and executive function similar to those seen in Alzheimer's disease (AD) have been reported (7). Unfortunately, clinical trials of anti-inflammatory agents have given conflicting and disappointing results, but studying specific stages of $A D$ have provided newer insights regarding the pathophysiology of pro- and antiinflammatory signaling (8). These insights demonstrate the urgent need to better understand the mechanisms of cellular activation and the concept of neuroinflammation in neurodegenerative disease more broadly.

HIV does not enter neurons, but rather the innate immune cells-resident microglia and macrophages in the brain become infected in a productive fashion, releasing new viral progeny. The resulting injury to the brain occurs through several indirect mechanisms which include dysregulation of glutamate metabolism and excitotoxicity, oxidative damage, and increased inflammatory signaling $(9,10)$. Microglia play a central role in surveying the health and integrity of neurons, and are responsible for maintaining a homeostatic CNS environment (11). Once activated, microglia transform from a functional resting state in which they appear highly ramified to more ameboid shapes with increased phagocytic activity and secretion of proinflammatory cytokines and chemokines that in turn activate other components of the neurovascular unit (12). The inability of microglia to return to a basal level of homeostatic surveillance is then postulated to underlie the progression of genetic or idiopathic neurodegenerative disorders which develop over time and manifest in people at older age $(13,14)$.

NSG immunodeficient mice reconstituted with human T-cells, monocytes and macrophages differentiated from engrafted hCD34 + hematopoietic stem cells provide the ability to study the cellular and molecular mechanisms by which HIV-induced inflammation disrupts neural functioning in the brain in a manner that is not possible in human clinical research (15-17). Furthermore, neuroimaging, in particular positron emission tomography (PET) is a critically important non-invasive translational tool that can identify neuropathologic inflammation as a consequence of a range of injuries to the brain, including neurodegeneration. Indeed, despite our incomplete understanding of the neuropathophysiology, a depth of experimental evidence supports correlations between increased expression of the translocator protein (TSPO) receptor on microglia (previously known as the peripheral benzodiazepine receptor) and the extent of neuroinflammation (18).

Previous in vitro, ex vivo $(19)$ and rhesus macaque studies $(20,21)$ have implicated a role for the multifunctional protein osteopontin (OPN) or secreted phosphoprotein-1 (SPP1) in proinflammatory signaling in HIV-associated neurocognitive disorders (HAND). Notably, the accumulating data points to several different underlying mechanisms, however the detailed mechanisms of OPN function remain to be clarified. Increased levels of OPN are found in the CSF and brains of HIV-infected persons with moderate to severe cognitive impairment (20-22). Cortical neurons treated with recombinant OPN were 
shown to be protected from HIV envelope-protein-induced damage to dendrites and axons in a dosedependent manner through mammalian target of rapamycin pathway signaling (mTORC1 and mTORC2) (23). Interestingly, OPN is also significantly elevated in the cerebrospinal fluid of individuals with Alzheimer's disease (AD), Parkinson's, multiple sclerosis and fronto-temporal dementia, but very little is known about its function in the brain in these contexts (reviewed in (24)).

To test whether OPN is required or not for HIV-induced CNS inflammation, we utilized buffer-injected, or HIV-infected NSG mice engrafted with human $\mathrm{CD} 34^{+}$together with weekly administration of RNAaptamers to inhibit global expression of OPN. Micro-PET-imaging of the mice with the TSPO ligand $\left[{ }^{11} \mathrm{C}\right] \mathrm{DPA}-713$ was performed at 12-weeks post-infection. Contrary to our hypothesis, we found that microglial activation was significantly increased in the cortex, hippocampus, olfactory bulb and other brain regions only in HIV-infected mice with decreased levels of OPN suggesting that OPN rather reduces neuroinflammation induced by HIV. Analyses of brain tissue by quantitative immunochemistry at the study endpoint demonstrated significant elevation in TSPO expression in microglia and in specific neuronal subsets with a quantitative relationship to OPN levels. An additional interaction between HIV replication and Iba- 1 expression was also found, suggesting that, in this experimental paradigm, there are at least two distinct pathways leading to neuroinflammation.

\section{Materials And Methods}

Study Design. The research objective was to test the hypothesis that osteopontin exacerbates HIV-

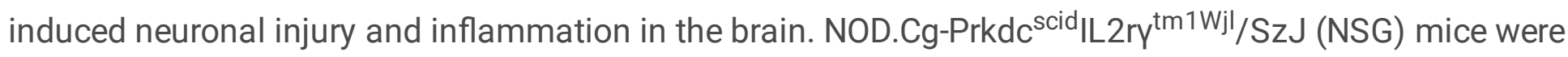
selected for this study as several publications demonstrated that this strain could be reproducibly engrafted with human immune cells and productively infected with HIV. Moreover, inflammation of the meninges and a small number of HIV-infected immune cells could be detected in the brain parenchyma (15). With $90 \%$ power to detect a $30 \%$ difference in our experimental variables (HIV, OPN) using a twosided test with alpha $=.05$, we determined that eight mice in each group would be required. NSG mice are susceptible to graft-vs-host disease and thus additional animals were included in the event that $\sim 5 \%$ of mice had to be euthanized. Animals (males and females were included equally in each grouping) were assigned to one of the four groups in a $2 \times 2$ treatment paradigm: two groups of TBS-injected animals served as uninfected controls and two groups were infected with $\mathrm{HIV}_{\mathrm{SF} 162}$, strain originally isolated from the brain of an HIV-infected individual with toxoplasmosis of the brain (as described below) (25). One group of TBS-injected, and one group of HIV-infected mice were intraperitoneally injected weekly with functional RNA aptamers ("OPN-R3"). The OPN-R3 aptamers blocked the interaction of osteopontin with its receptors and stimulate a feedback loop that reduces gene and protein expression (26). The second groups of TBS-, or HIV-infected animals received mutated aptamers ("mutant OPN-R3") with sequence alterations that prevent engagement of OPN with aptamer receptors and thus, served as negative controls. The animal handlers were not blinded to each group due to the biosafety level 2 requirements of working with HIV-infected mice. 
Generation of humanized mice. The animal protocol and all procedures were reviewed and approved by the Johns Hopkins University Animal Care and Use Committee and Institutional Review Board. Male and female NSG mice (5-8 weeks old) were purchased from Jackson Laboratory and maintained under pathogen-free conditions. Mice were first acclimated to the suite with automatic watering system for two weeks before monogamous pairing. Mice had free access to food and water and were housed in automatically controlled light conditions (light 7 am-9 pm). To avoid secondary microbial infections of these immunocompromised mice, cages were changed in a vented biosafety cabinet. All work surfaces, as well as cages, were thoroughly sanitized with chlorohexidine solution. Two-day old pups were irradiated with $100 \mathrm{cGy}$ using a Gamma Cell-40 Cesium Extractor (Theratronics) followed by sedation with isoflurane in an induction chamber. The sedated pups were subjected to intrahepatic injection of 1$2 \times 10^{5}$ cells/mouse of human CD34+ HSCs in 50-60 $\mu$ of sterile PBS with a 30-gauge needle (HSCs from cord blood from STEM Cell, Inc. or fetal liver from Advanced Bioscience Resources using magnetic bead separation, Miltenyi Biotec, 113-117-043). The engrafted pups were weaned at 3-4 weeks of age and males and females housed as separate groups. Peripheral blood $(\sim 50 \mu \mathrm{l})$ taken by facial bleed was sampled at regular intervals to quantify human leukocytes by seven-color flow cytometry with the following antibodies in a protocol optimized for small mouse blood volumes: human pan-CD45-Viogreen or PerCP (Miltenyi Biotec, 130-110-638, ThermoFisher MHCD4531), CD3-PE (ThermoFisher, MHCD0304), CD4-Vioblue (Miltenyi Biotec,130-113-219), CD8-APC-Vio770 (Miltenyi Biotec, 130-113-155), CD14-APC (BioLegend, 301808), CD19-PerCP (eBiosciences, 11-0199-42; BioLegend, 302228) and mouse CD45-FITC (eBiosciences,11-0451-82). Three to four months after engraftment, mice were sedated with isoflurane and intravenously injected with $1 \times 10^{5}$ tissue culture infectious doses/ml of $\mathrm{HIV}_{\mathrm{SF} 162}$ in $\sim 50 \mu \mathrm{l}$ or with TBS buffer using a 25-gauge needle. The HIV-infected and buffer-injected mice were housed in separate same-sex social groups (when possible).

Treatment with inhibitory aptamers. Two weeks after infection, mice were injected (intraperitoneal) with $1 \mathrm{mg} / \mathrm{kg}$ of HPLC-purified RNA aptamers against OPN (IBA Lifesciences). The sequences for the aptamers were: mutant OPN-R3 (CGG-CCA-CAG-AAU-GAA-UCA-UCG-AUG-UUG-CAU-AGU-UG) and OPN-R3 (CGG-CCA-CAG-AAU-GAA-AAA-CCU-CAU-CGA-UGU-UGC-AUA-GUU-G). Both aptamers were modified at the 2'-O position with a methyl group, and the OPN-R3 aptamer included phosphorothioate modified bases (PTO) to help protect the nucleic acids from rapid attack by endo- and exonucleases (as given in reference (26). HPLC-purified lyophilized aptamers were refolded by resuspension in Folding Buffer $(1 \mathrm{x}$ PBS $8 \mathrm{mM}$ sodium phosphate dibasic; $2 \mathrm{mM}$ potassium phosphate monobasic;137 mM NaCl; pH 7.4,) with $1 \mathrm{mM} \mathrm{MgCl} 2$, heated at $95^{\circ} \mathrm{C}$ for 5 minutes ( $\mathrm{min}$ ) and allowed to cool to room temperature for $15 \mathrm{~min}$ before use. For the PTO modified OPN-R3 aptamer, an additional reduction step was required and performed using Tris(2-carboxyethyl)phosphine (TCEP) disulfide reducing gel slurry (ThermoScientific). A volume of TCEP slurry equal to the volume of aptamer solution was added to a microfuge tube with 3 volumes of Folding Buffer, vortexed then centrifuged for $1 \mathrm{~min}$ at $1000 \times \mathrm{g}$ and the supernatant discarded. This step was repeated. The cooled aptamer from above was added to the washed slurry, vortexed briefly and the tube allowed to incubate at room temperature for $10 \mathrm{~min}$ with mixing after the 
first $5 \mathrm{~min}$. The tube was centrifuged as above and the supernatant containing the reduced aptamer collected into a clean microfuge tube for use as described above.

HIV Viral Load Quantification. RNA was isolated from 56 mice using QIAamp Viral RNA mini kit (Qiagen) and concentrations determined using NanoDrop 2000 (Thermo Scientific). Samples of insufficient quality were treated with DNase to remove contaminating DNA (Life Technologies, Carlsbad, CA), followed by ethanol precipitation to concentrate any samples with less than $10 \mathrm{ng} / \mu \mathrm{l}$ of RNA. Samples were mixed with $1 / 10$ th volume of $3 \mathrm{M}$ sodium acetate, 2.5 volumes of ice-cold $100 \%$ ethanol, and $1 \mu \mathrm{I}$ of $20 \mathrm{mg} / \mathrm{ml}$ glycogen, and kept at $-80^{\circ} \mathrm{C}$ for one hour. Samples were then centrifuged at $4^{\circ} \mathrm{C}$ for 30 minutes, followed by two $70 \%$ ice-cold ethanol washes of the pellet. Pellets were resuspended in the appropriate volume of sterile, deionized water. RNA of insufficient concentration and quality were not used in the final results (7 out of 47 total samples). RNA was reverse transcribed in a $20 \mu \mathrm{l}$ reaction using $50 \mathrm{ng} / \mu \mathrm{l}$ of random hexamers, $10 \mathrm{mM}$ dNTP mix, 5X First-Strand Buffer, 0.1M DTT, RNaseOUT recombinant RNase Inhibitor, $10 \mathrm{pg}-5 \mu \mathrm{g}$ of RNA, sterile water, and SuperScript III reverse transcriptase (Invitrogen/ThermoFisher). For Taqman qPCR, a custom design was optimized using forward primer Seq162F: 5'CGA-ACC-CAG-ATT-GTAAGA-CT, reverse primer Seq162R: 5'ACA-TGC-TGT-CAT-CAT-TTC-TTC and probe: 5'-FAM/AG-CAT-TAGG/ZEN/A-CCA-GCA-GCT-ACA-CT/3IABKFQ (Integrated DNA Technologies). Amplification was performed with TaqMan Fast Advanced Master Mix, with conditions as follows: UNG incubation $50^{\circ} \mathrm{C}, 2 \mathrm{~min}$, one cycle; enzyme activation, $95^{\circ} \mathrm{C}, 20 \mathrm{sec}$, one cycle; 40 cycles of denature $95^{\circ} \mathrm{C}$, one sec, anneal, $48^{\circ} \mathrm{C}, 4 \mathrm{sec}$; extension, $60^{\circ} \mathrm{C}, 16 \mathrm{sec}$ QuantStudio 3 (Applied Biosystems). RNA isolated from viral particles (HIV-1 SF162) were used to create a 10-fold dilution standard curve performed on the CDNA with a lower detection limit of 1.0 copies $/ \mathrm{ml}$. The slope was -3.759 and the $R^{2}$ value was 0.995 , which both fell within the acceptable range for real-time PCR assays.

Micro-Positron Emission Tomography (PET) imaging. Animals to be imaged were moved to the small animal PET imaging room at least 1 hour before the experiments commenced. A dedicated small animal PET scanner (eXplore VISTA; GE Healthcare) and small animal CT scanner (X-SPECT/CT; Gamma Medica) were used. In all experiments, the PET scan was conducted for two mice simultaneously with the same batch of $\left[{ }^{11} \mathrm{C}\right] \mathrm{DPA}-713$. Batches of [11C]DPA-713 were synthesized at high specific activity and radiochemical purity at the Johns Hopkins PET Radiotracer Center according to the literature (27). One female mouse from a pair represented a control group $\left(\right.$ Buffer-OPN ${ }^{+-}$) whereas the other female mouse represented an experimental group (HIV-infected-OPN ${ }^{+/-}$). Mice were anesthetized with isoflurane and catheterized and PET-imaging began immediately after an intravenous bolus injection of the tracer. For the quantitative analyses of uptake intensity, the following tracer parameters were recorded: mouse weight, tracer specific activity, amount of tracer injected, amount of tracer remaining in the syringe, time at which residual was measured, and start time of scan. Dynamic PET scans were acquired for $60 \mathrm{~min}$ ( $20 \sec \times 3,30 \sec \times 2,1 \mathrm{~min} \times 2,2 \mathrm{~min} \times 3,5 \mathrm{~min} \times 10)$ and a CT scan was acquired immediately after for the purpose of localizing brain regions as described below. The individual performing the analyses were blinded to the identity of the experimental groups. PET images were reconstructed using an iterative 2D ordered-subject expectation-maximization method, using a trans-axial pixel size of $0.39 \mathrm{~mm}$ and axial 
slice thickness of $0.78 \mathrm{~mm}$ [1]. No attenuation and scatter corrections were applied, as they have relatively small impact on mouse brain imaging. Image analysis was performed using the PMOD software package (v3.7, PMOD Technologies Ltd, Zürich, Switzerland). For each study, the reconstructed PET and CT brain images were first co-registered; the existing Mouse Brain Template Volumes of Interest (VOIs) [2,3] were then "morphed" to match the brain image of the fused PET-CT; finally, the pre-defined VOls were then applied to the dynamic PET data to generate time-activity curves (TACs) in the unit of percent injected dose per gram of tissue $(\% \mathrm{ID} / \mathrm{g})$.

Tissue harvest. Mice were deeply anesthetized with isoflurane, then subjected to intracardiac puncture for terminal blood collection followed by perfusion with cold PBS buffer. The brain was isolated from the skull intact, cut along midline in the sagittal plane into two halves, with one fixed in $4 \%$ paraformaldehyde for $\sim 24 \mathrm{hrs}$ and later transferred to $70 \%$ ethanol before further processing for paraffin embedding and sectioning into $5 \mu \mathrm{m}$ slices (Johns Hopkins Path Services). The remaining half of brain was placed in RNAlater (ThermoFisher) overnight and frozen within $18-24$ hrs at $-80^{\circ} \mathrm{C}$ before RNA extraction. RNA from the brain was isolated using Monarch Total RNA Miniprep Kit (New England Biolabs) and concentrations were determined using NanoDrop 2000 (Thermo Scientific). Each brain sample was thawed from $-80^{\circ} \mathrm{C}$ and $30 \mathrm{mg}$ slices were homogenized with $600 \mathrm{uL}$ of 1X DNA/RNA protection reagent. $60 \mathrm{uL}$ of Protease $\mathrm{K}$ reaction Buffer and $30 \mathrm{uL}$ of Protease $\mathrm{K}$ were added, and then the tube was vortexed. Each sample was incubated at $55^{\circ} \mathrm{C}$ for 7 minutes, followed by the addition of one volume of lysis buffer. Samples were vortexed well, and then transferred to a gDNA Removal Column. After spinning down for 30 seconds at $16,000 \times \mathrm{g}$, the elute was combined with one volume of $100 \%$ ethanol and mixed by pipette. The mixture was transferred to an RNA purification column and spun for 30 seconds. The speed remained at $16,000 \times \mathrm{g}$ for all centrifugation steps. After the first spin in the RNA purification column, the flow through was discarded and extra RNA enhancement steps were performed as described in the Monarch Total RNA Miniprep Kit. Following these extra RNA enhancement steps, 500uL of RNA Priming Buffer was added to the column and spun for 30 seconds. Two washes with RNA Wash Buffer were preformed, the second lasting for 2 minutes instead of 30 seconds. Finally, the column was transferred to a sterile $1.5 \mathrm{~mL}$ RNase free microfuge tube and eluted with $60 \mathrm{uL}$ of nuclease free water by a 30 second spin.

Immunohistochemistry. After warming for $10 \mathrm{~min}$ at $60^{\circ} \mathrm{C}$, brain sections were deparaffinized in a $100 \%$, $95 \%, 70 \%$ alcohol series with final rehydration in $1 \mathrm{X}$ tris-buffered saline-TBS $(0.02 \mathrm{M}$ Tris- $\mathrm{HCl}, 0.15 \mathrm{M}$ $\mathrm{NaCl}, \mathrm{pH} 7.4$ \#351-086-101). Sections were incubated with proteinase K (Electron Microscope Sciences) at $37^{\circ} \mathrm{C}$ for 20 min then immersed in citrate buffer $\mathrm{pH} 6.0$ at $99^{\circ} \mathrm{C}$ for $10 \mathrm{~min}$ then allowed to cool at room temperature for $20 \mathrm{~min}$. Slides were placed in 1X TBS then treated for 10 min with Bloxall (VectorLabs) and $3 \%$ hydrogen peroxide to inactivate tissue peroxidases and alkaline phosphatases (AP). Slides were then incubated with $2 \%$ Donkey sera/ $0.3 \%$ Triton X-100, $0.1 \%$ Tween-20 for one hour at room temperature, followed by blocking with Donkey anti-mouse $F_{a b}$ (1:25; \#715-007-003, Jackson ImmunoResearch Laboratories, Inc) for 1 hour. Sections were incubated with antibodies at $4^{\circ} \mathrm{C}$ for at least $16 \mathrm{hrs}$. For double labeling, staining for TSPO was performed first followed by detection of Iba-1. Antibodies used: 
goat anti-mouse OPN (R\&D Systems \#AF808; tissue blocked in 10\% rabbit serum/0.3\% Triton X-100, 0.1\% Tween-20), mouse anti-TSPO (1:100, MA5-24844, Invitrogen/ThermoFisher), mouse anti-lba-1 (MA527726, Invitrogen/ThermoFisher), anti-tyrosine hydroxylase (1:500, \#Ab112, Abcam). Slides were rinsed in 1X TBS then incubated with 1:500 dilution of donkey anti-mouse secondary antibodies conjugated to AP (\#AP15999-anti-goat, MilliporeSigma, \#715-056-151-anti-mouse, Jackson ImmunoResearch Laboratories, Inc) or horseradish peroxidase (HRP) (\#715-036-150, Jackson ImmunoResearch Laboratories, Inc). Slides were developed with the Vector Immpact Red and DAB kits (\#SK-5105, \#SK4105, Vector Labs) followed by dehydration and mounting in Cytoseal 60 (\#8310-4 Richard-Allan Scientific, ThermoFisher). Images were captured on a Zeiss Axio Observer A1 inverted microscope (20x objective), and a digital copy of the raw image was adjusted in the same manner for each, for optimal brightness and contrast, using Adobe Photoshop CS5.1. Staining intensity was quantified using ImageJ/Figi 2.0. A binary image was created and the threshold for the maximum and minimum intensity values in each channel applied. The pixel area was calculated using the measure function.

HIV-1 RNA in situ hybridization with RNAscope. Chromogenic RNA in situ hybridization was performed

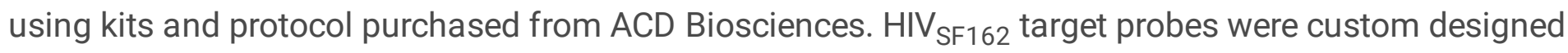
to target Env-Nef sequences obtained from the accession number M65024.1 (2621-3701). PFA-fixed paraffin embedded brains and spleens were sectioned $(10 \mu \mathrm{M})$ and placed on SuperFrost Plus slides (Johns Hopkins Path services). Slides were baked in a slide oven for 1 hour at $60^{\circ} \mathrm{C}$ prior to deparaffinization. Slides were incubated twice in Histoclear for 5 minutes and washed in $100 \%$ ethanol twice for 1 minute. Sections were incubated in Bloxall (VectorLabs) for 10 minutes at room temperature to quench endogenous peroxidases. Slides were then washed with distilled water and moved to a steamer containing 2 slide incubation chambers. One contained distilled water and the other contained $1 \mathrm{X}$ RNAscope target retrieval buffer heated to at least $99^{\circ} \mathrm{C}$. Slides were temperature adjusted by gently agitating them for 10 seconds in the distilled water chamber and then moved to target retrieval buffer and allowed to boil for 15 minutes. Slides were then washed in distilled water and transferred to $100 \%$ ethanol for 3 minutes and then allowed to dry. Hydrophobic barrier pen was used to make a boundary around the tissue. Proteinase $\mathrm{K}$ treatment was applied to the tissues and incubated in a HybEZ oven at $40{ }^{\circ} \mathrm{C}$ for 15 minutes. Slides were washed with distilled water and incubated with pre-warmed target probes for 2 hours in the HybEZ oven at $40^{\circ} \mathrm{C}$. Slides were saved overnight in in $5 \mathrm{X}$ SSC buffer. Further amplication of target probe signal was performed the following day according to the manufacturer's instructions (RNAscope 2.5 HD detection protocol). Amp 1 was applied and incubated in HybEZ at $40{ }^{\circ} \mathrm{C}$ for 30 minutes. Slides were washed in RNAscope wash buffer and treated with Amp 2 and incubated in HybEZ at $40{ }^{\circ} \mathrm{C}$ for 15 minutes. Following another wash Amp 3 was applied and incubated in HybEZ at $40^{\circ} \mathrm{C}$ for 30 minutes. Amp 4 was applied after another wash and incubated in HybEZ at $40{ }^{\circ} \mathrm{C}$ for 15 minutes. Amp 5 was applied following another wash and incubated at room temperature for 30 minutes. Amp 6 was applied before signal detection and incubated at 15 minutes at room temperature. Fast red was detected by combining Red-A and Red-B (1:60) and added to the sections which developed in 10 minutes at room temperature. The slides were counterstained with 30\% Gill's Hematoxylin I (MilliporeSigma GHS132) and 
allowed to dry on a $60^{\circ} \mathrm{C}$ slide warmer for 15 minutes. Slides were mounted with Cytoseal 60 (\#8310-4 Richard-Allan Scientific, ThermoFisher) and imaged on ECHO Revolve microscope at 40x magnification.

Statistical analyses. Data were analyzed using ordinary one-way ANOVA and subsequent Tukey's test for multiple comparisons or Kruskal-Wallis test, followed by Dunn's multiple comparison test (non-parametric data). To determine whether there was any interaction between knockdown of OPN or not and HIV infection, data were also analyzed by two-way ANOVA with Spearman's test for heteroscedasticity, and normality of residuals (Anderson-Darling, D'Agostino-Pearson omnibus as \pm standard deviation (SD), and a minimum $p$ value of 0.05 was estimated as the significance level for all tests (GraphPad Prism 8). ANOVA two-tailed Student's $t$ test and significance $(P<.05)$.

\section{Results}

\section{A. A model of chronic low-level HIV persistence in CD34 ${ }^{+}$ humanized mice}

Despite the lack of robust HIV replication in the brain of NSG-hCD34 mice, which lack human microglia, productive replication in human target T-cells and myeloid cells in the CNS and periphery induces microgliosis, astrocytosis and alterations in neuronal metabolites (15). We adapted this model to test the hypothesis that osteopontin (OPN) also known as secreted phosphoprotein-1 (SPP1), is required for HIVmediated inflammation in the central nervous system (CNS). The experimental design included four groups composed of male and female mice engrafted with human $\mathrm{CD} 34^{+}$hematopoietic stem cells (hCD34, HSCs), which differentiate into mature human T-cells and macrophages that permit infection with HIV. Two groups were intraveneously inoculated with buffer, and the other two groups with $\operatorname{HIV}_{\mathrm{SF} 162}$. After two weeks, mice were injected weekly with either functional (HIV OPN ${ }^{-}$) or mutant aptamers (HIV $\mathrm{OPN}^{+}$) that do not block OPN expression (26). We make note that due to 1) the capability to conduct microPET-imaging on two mice at a time and 2) the need to synthesize the radiotracer fresh each time because of its short-half-life, we had to limit the number of mice that could be imaged and given these limitations, decided to test only females based on a recent publication suggesting sex differences in HIV CNS disorders (28). We also note that fewer data points at earliest time periods after infection. We discovered weeks later that the efficiency of viral RNA recovery after low temperature freeze/thaw of mouse blood was inefficient and needed to be performed within $24 \mathrm{hrs}$ after collection. Quantitative PCR analyses of virus release into the plasma at 10 and 16-weeks after aptamer treatment revealed no significant differences between experimental groups (Fig. 1SA-B). However, analyses by two-way ANOVA demonstrated that time accounted for $20 \%$ of the variance between the viral loads at the 10 - and 16 -week time points ( $\mathrm{n}=3 \mathrm{HIV}-\mathrm{OPN}+, \mathrm{n}=8 \mathrm{HIV}-\mathrm{OPN}-, P=.0369)$. Similarly, analyses of virus release into the plasma over a period of 16 weeks (112 days) after infection revealed a wide variation in replication levels among individual female mice and overall, no significant differences among the two groups were detected (Fig. 1A). However, in HIV OPN ${ }^{+}$mice, replication increased from the initiation of aptamer treatment until the last sampling point at 98 days after infection, a trend that was less apparent in the HIV 
$\mathrm{OPN}^{-}$group (Fig. 1B). During this same time period, flow cytometric analyses of circulating white blood cells in the plasma revealed a gradual and stark decline of hCD45 cells in the HIV OPN ${ }^{+}$, which contrasted with the higher levels detected in the HIV OPN ${ }^{-}$group, however the differences in slopes were not quite significant (Fig. 1C, $\mathrm{F}=3.800$. DFn $=1, \mathrm{DFd}=17, P=0.0680$ ). While a trend in the decrease in the absolute percentage of hCD4 cells was similar in both groups, over time the decline in hCD8 cells in the plasma of HIV OPN ${ }^{-}$was less compared to the HIV OPN ${ }^{+}$group, but neither trends were significant (Fig. 1D-E, CD4, F $=0.3051 . \mathrm{DFn}=1, \mathrm{DFd}=17, P=0.5879 ; \mathrm{CD} 8, \mathrm{~F}=1.489 . \mathrm{DFn}=1, \mathrm{DFd}=17, P=0.2390$ ). Therefore, we surmised that the detection of virus replication in the plasma at the 16-week study endpoint was likely due to a combination of virus release from productively infected circulating immune cells and tissue reservoirs. To detect HIV RNA (vRNA) expression in the brain and other tissues, we used in-situ hybridization or RNAscope. Large foci of vRNA positive cells were readily detected in spleen, a tissue rich in HIV target T-cells and macrophage populations (Fig. 1F). In mice with the highest viral loads, vRNA near the brain vasculature and in the parenchyma in both the infected aptamer treated groups were detected, but infrequently (Fig. 1F).

To ascertain the effectiveness of the aptamers in reducing OPN gene expression, the entire gastrointestinal (GI) tract collected at the study endpoint was immunostained for OPN protein. Because the GI tract is the largest tissue system in the body, it was used as a proxy for assessing the efficiency of the aptamer treatment. While there were no differences in OPN reactivity between the buffer treated groups, significantly elevated staining was detected in the small intestines of HIV OPN ${ }^{+}$mice in contrast to infected mice injected with functional aptamers (HIV OPN ${ }^{-}$group) (Fig. 1G \& H). This difference in reactivity was influenced by highly significant interactions with HIV (two-way ANOVA, $P<.0001$ ) and OPN levels (two-way ANOVA, $P=.0003$ ) (Fig. 1 H, right graph). Quantitative RT-PCR analyses on RNA purified from brain tissue showed a trend of decreased message in both the buffer OPN ${ }^{-}$and HIV OPN ${ }^{-}$mice treated with functional aptamers, but the differences did not reach statistical significance (Fig. 1I).

\section{B. Increased uptake of TSPO ligand in mice with decreased osteopontin (OPN) expression To determine} the impact of chronic HIV infection in the presence of normal or lowered OPN levels on microglial activation in live mice, female animals in each group were anesthetized and subjected to PET-imaging using the ligand [11C]DPA-713 at 12 weeks post infection (27). Females were chosen because, over the course of the study, anecdotal differences in their anxiety compared to male mice was observed. DPA-713 ligand binds to the translocator receptor (TSPO) expressed prominently on activated microglia (29), and to a lesser extent, on the endothelium, astrocytes, and neurons of the dorsal root ganglion (30).

Significant differences in DPA-713 uptake between the two HIV-infected groups treated with functional (HIV OPN ${ }^{-}$) or mutated aptamers (HIV OPN ${ }^{+}$) and both buffer control groups in the 20-50 min time window after bolus injection were observed across the entire brain (Fig. 2A-C). Interestingly, the highest levels of uptake of DPA-713 ligand amongst the four experimental groups were seen in the HIV OPN ${ }^{-}$ group (Fig. 2B-C). Analyses of specific brain regions showed significantly increased ligand uptake in the hippocampus, striatum, olfactory bulb, cortex, thalamus, cerebellum, amygdala, central grey matter, midbrain, basal forebrain septum, hypothalamus, and in the inferior and superior colliculi of HIV OPN ${ }^{-}$ 
mice compared to the other experimental groups (Fig. 2D-R). In the right striatum, olfactory bulb, cortex, and basal forebrain in particular, mice in the HIV OPN ${ }^{-}$group that had the highest viral loads also showed the greatest uptake of DPA-713 ligand, suggesting a direct relationship between the extent of virus replication, and the amount of TSPO binding (Fig. 2E-G, 2N). Interestingly, in these regions, the uptake of label was the highest and sustained over time (Fig. 2 Supplemental, F, G, H, Q). In two regions, the right striatum and right amygdala, the HIV OPN ${ }^{+}$group had more ligand uptake than the buffer $\mathrm{OPN}^{-}$group (Fig. 2E, 2J). Analyses of time-activity curves and the early 8-10 min window after bolus injection did not show any significant differences in the amygdala among the experimental groups in DPA-713 uptake (Fig. 2Supplemental, K), which was in contrast to the 20-50 min window (Fig. 2J). It was notable that ligand uptake in the cortex and olfactory bulb was relatively fast and sustained over the $1 \mathrm{hr}$ imaging period (Fig. 2 Supplemental, G-H).

\section{TSPO-Iba-1 immunoreactivity is abundant in cortical and hippocampal regions}

PET imaging with ligands that detect microglial cell activation as an indirect measure of neuroinflammation has been an active area of research as it allows insight into neuropathologic processes in living persons. Recent findings have noted that cells other than microglia can upregulate TSPO expression (31). To determine whether increases in TSPO uptake detected by PET-imaging in these mice correlated with measures in brain tissue at the 16-week study endpoint, double-label IHC was performed to detect cells labeling for $\mathrm{TSPO}^{+}$(red color) and/or Iba- $1^{+}$microglia (brown color). There are relatively few published studies that have analyzed TSPO expression in brain tissue in conjunction with neuroimaging (31-34). We first conducted a qualitative survey of brain sections from each group of mice. Abundant TSPO reactive neurons and microglia of variable intensities were seen in the cortex (Fig. 3A, representative shown, Buffer $\mathrm{OPN}^{+}$). TSPO ${ }^{+}$Iba- ${ }^{+}$cells having ramified or dystrophic phenotypes were readily detected in the midbrain (Fig. 3B, representative shown, HIV OPN+). In both HIV-infected groups (Fig. 3C iii and iv) the organization of the dentate gyrus appeared altered compared to the buffer control groups (Fig. 3C i and ii). Additionally, there were more cells within hippocampal fissures in the HIV$\mathrm{OPN}^{+}$group compared to the other three groups (Fig. 3Ciii, red circles). TSPO labeling was strong within the hippocampal neurons and fissures and in a small number of mice, reactivity in the meningeal layer was also found (Fig. 3D-G). In contrast to the ordered array of Purkinje neurons of the cerebellum in HIV$\mathrm{OPN}^{+}$mice (Fig. 4A-C), in the HIV-OPN ${ }^{-}$group, the intensely stained TSPO+ Purkinje neurons displayed a disordered pattern (Fig. 4E) that was not seen in the Buffer-OPN ${ }^{-}$group (Fig. 4D). Moreover, a select subset of neurons, which we suspected were located in the substantia nigra, reacted very strongly with TSPO antisera (Fig. 5A-F). The identity of this brain region as the substantia nigra region was confirmed by the specific reactivity of these neurons individually with antisera against either TSPO or tyrosine hydroxylase (Fig. $5 \mathrm{H}-\mathrm{I})$.

\section{E. Iba-1 but not TSPO immunoreactivity is increased with osteopontin (OPN) suppression For post-} mortem immunohistochemistry and quantitative analyses, brains from mice that were subjected to neuroimaging were included in the analyses (Buffer-OPN ${ }^{+}, \mathrm{n}=3$; Buffer-OPN ${ }^{+}, \mathrm{n}=5 ; \mathrm{HIV}^{-O P N}{ }^{+}, \mathrm{n}=3$; HIV- 
$\mathrm{OPN}^{+}, \mathrm{n}=3$ ). In the HIV-OPN ${ }^{-}$group, one mouse died before necropsy and another from that group which was not imaged, was included to have $n=3$. Quantitative analyses of TSPO antibody reactivity (one-way ANOVA with Krusal-wallis test, Dunn's test) showed significantly reduced intensity in the buffer-OPN ${ }^{-}$ $(8.62+/-5.24)$ compared to the buffer $(13.73+/-8.24)$ and HIV OPN ${ }^{+}(13.7+/-7.8)$ groups (Fig. $6 \mathrm{~A}, \mathrm{P}$ $=.0087$ and $P=.003$, respectively). Interestingly, while no significant differences in TSPO

immunoreactivity between the HIV-infected groups were detected, the presence or absence of OPN was a significant interacting factor $\left(P=.0136, F(1,190)\right.$, Fig. 6B). The HIV-OPN ${ }^{-}$group showed significantly elevated Iba-1 immunoreactivity compared to uninfected mice with normal expression of OPN (Fig. 6C, $p$ $=. \mathbf{0 0 7 4}$, one-way ANOVA, Kruskal-Wallis, Dunn's test). Perhaps due to the lower number of mice available for the analyses, the increase in Iba-I immunoreactivity did not reach statistical significance, between the HIV OPN ${ }^{+}$and HIV OPN ${ }^{-}$groups (Fig. 6C). Nevertheless, viral infection significantly drove the increased level of Iba-I immunoreactivity in the brain (Fig. 6D, two-way ANOVA $p=.0051)$. Collectively, these results suggest that HIV-infection increases the number of activated microglia in the brain, while the level of OPN in this compartment had a greater impact on TSPO expression.

\section{Discussion}

Knowledge that there is continuous monitoring of proper neuronal function and integrity by microglia, the resident immune cells in the CNS, fundamentally altered the way in which we think about the role these cells play in health and disease $(35,36)$. Significant disruptions of the homeostatic brain

microenvironment, whether secondary to injury, neurodegeneration or viral infection, dramatically activate microglia. The result is upregulation of gene expression, morphologic conversion to a functional phagocytic phenotype, and the elaboration of pro- and anti-inflammatory mediators (37). Interestingly, recent detailed transcriptomic analyses of microglia from humans with neurodegenerative disorders, and the corresponding mouse disease models, revealed a common gene signature which includes osteopontin (OPN/SPP1) as a significantly enriched expressed gene in so-named "neurodegenerative" microglia (38-40). Evidence that OPN is significantly elevated in the plasma and/or CSF of people with neurodegenerative disorders and in HIV-associated cognitive impairment or HAND, has been reported (24). Prior studies have examined a role for OPN in protecting neurons after injury and stroke, but few have examined its potential functional impact on microglia (41). Our prior studies implicated OPN as an upregulated protein in HIV-infected macrophages that also stimulates viral replication through a NF-kbdependent mechanism (22). We went on to demonstrate, using post-mortem brain tissue from individuals with HAND, that neurons as well as microglia, show elevated OPN expression (19). OPN knockout mice are viable and display no gross physical or behavioral abnormalities but do show disorganized wound remodeling and defective macrophage infiltration after injury or infection $(42,43)$. This led us to our current study to determine whether or not OPN is required for HIV-mediated neuroinflammation in the brain.

TSPO, formerly known as the peripheral benzodiazepine receptor, is a small $18 \mathrm{kDa}$ protein found in the outer membrane of mitochondria whose expression is significantly increased in the brain after injury and 
neuroinflammation (reviewed in (18)), however there is no consensus about its exact physiologic roles (18). The availability of effective tools to clinically evaluate HIV-infected persons for neuropathologic evidence of cognitive impairment by non-invasive methods has been a critical focus of the field (29). TSPO PET-imaging with first-generation ligands showed promise in both the rhesus macaque models of HIV brain disease (44) and in humans (45). Imaging with the second-generation ligand DPA-713 revealed significant abnormalities in the frontal cortex of HIV-infected persons with severe cognitive impairment (29). A more recent association study found positive correlation between increased DPA-713 uptake and neurocognitive performance in treated-HIV-infected persons in line with prior human imaging studies (28, 46-48).

The microPET-imaging results from this study strongly suggests that, rather than potentiating HIV injury, OPN acts as a molecular brake helping to dampen the microglial inflammatory response. In this regard, TSPO levels quantified by $\mathrm{IHC}$ were higher in $\mathrm{OPN}^{+}$compared to $\mathrm{OPN}^{-}$mice, with significant increases seen in the buffer-injected group. Moreover, additional analyses revealed an interdependence between TSPO and OPN levels that warrants further investigation to understand the disconnect between our neuroimaging and IHC findings. The detection of TSPO expression in cortical, Purkinje and striatal neurons by IHC, suggests that these cells also contribute to the overall inflammatory state in the brain, but the underlying neurobiological basis for this finding is unknown (18). In this regard, a recent report describes low-level basal expression of TSPO in several regions of the brain including vascular endothelial cells and in Purkinje neurons in normal mouse brain compared to tissues from a TSPO knockout mouse (33). Moreover, TSPO can also be expressed in astrocytes, and these cells were not assessed in this study. Early intraneuronal accumulation of toxic amyloid in a mouse model of Alzheimer's disease was recently shown to initiate inflammatory gene expression of chemokines CCL2 and CCL3 in pyramidal neurons of the hippocampus, thus demonstrating that neurons have the capacity to initiate such signaling (49). Clearly, additional studies are needed to have a more complete understanding of the neuroinflammatory response.

In contrast, for Iba-1, a well-established marker for microglial activation, elevation was dependent on the extent of HIV replication. This finding is in alignment with early neuropathological findings of microgliosis in post-mortem brains of HIV-infected individuals (50). The association of activated microglia and increased secretion of proinflammatory cytokines in plasma and CSF are neuropathologic hallmarks in HAND (50). However, with antiviral treatment, cellular activation related to ongoing virus replication is greatly diminished, though not completely eliminated (51). Moreover, our findings demonstrate that there may be two or more different signaling pathways targeting microglia, TSPO and/or Iba-1, which are activated by HIV infection and could potentially explain why, in TSPO knockout mice, microglia remained, able to respond appropriately to neuronal injury (32).

Ruling out underlying genetic susceptibility to dementia, there are competing ideas, supported by data suggesting that cognitive deficits could be related to a legacy effect (52) or low-level chronic HIV replication in the brain (53), both of which would inflict accumulating damage to neural networks over the span of time. Our mice model in which HIV RNA expression was detected in the brain in only a few cells 
per five-micron section represents the latter, low-level chronic infection modeling approximately 48-55 human years. Nevertheless, only by the disruption of OPN function could we detect by microPET-imaging the profound impact HIV has on promoting neuroinflammation.

\section{Conclusions}

Although OPN has diverse functions in different physiological and pathological processes, much evidence supports it having a central role in inflammatory signaling (24). It was identified as a highly phosphorylated protein purified from osteoclasts, which are the macrophages of the bone $(54,55)$, but it can also be made by T-cells and fibroblasts (24). Mice express a single OPN allele. In contrast, human cells can express one or more of three splice variants of OPN, leading to an intracellular or extracellular form of protein which is further subject to proteolytic cleavage that can generate peptides possessing signaling activity (reviewed in (24)). However, the significance of these variants in regulating neuroinflammation has not, to our knowledge, been studied in the CNS. Our findings suggest that a deeper understanding of OPN-regulation of innate immune signaling of neuroinflammation would be extremely valuable in the search for novel approaches which are urgently needed to promote the return to homeostasis in the CNS microenvironment in HAND and other neurocognitive disorders.

\section{List Of Abbreviations}

$\begin{array}{ll}\text { AD } & \text { Alzheimer's disease } \\ \text { CNS } & \text { central nervous system } \\ \text { HAND } & \text { HIV-associated neurocognitive disorder } \\ \text { HIV-1 } & \text { human immunodeficiency virus type-1 } \\ \text { IBA-1 } & \text { ionized calcium-binding adaptor molecule-1 } \\ \text { IHC } & \text { immunohistochemistry } \\ \text { MTORC } & \text { mammaliam target of rapamycin } \\ \text { NSG } & \text { NOD.Cg-Prkdc }{ }^{\text {scid } / L 2 r g}{ }^{\text {tm } 1 \text { Wjl } / S z J} \\ \text { OPN } & \text { osteopontin } \\ \text { PET } & \text { positron emission tomography } \\ \text { SPP1 } & \text { secreted phosphoprotein-1 } \\ \text { TSPO } & \text { translocator protein }\end{array}$




\section{Declarations}

Ethics approval for research involving animals. Johns Hopkins University is fully accredited by the Association for the Assessment and Accreditation of Laboratory Animal Care that shares foundational principles articulated in the Basel Declaration. The research protocol was approved by the Johns Hopkins Institutional Review Board and the Animal Use and Care Committee and conducted using biosafety level 2 best practices.

Consent for publication. All authors contributed to the editing of the manuscript and have consented for publication.

Availability of data and materials. All data generated and analyzed during this study are included in this article and its supplementary information files.

Competing interests. All authors declare that they have no competing interests.

Funding. This study was supported by funding from the USA, National Institutes of Neurological Disorders and Stroke (NINDS), R01NS102006 to A.M.B.

Authors' contributions. Author contributions: F.J.M., A.M.B. and E.G. generated humanized mice and performed injections. E.G. performed RNA isolations and performed qPCR analyses. F.J.M., and A.M.B., prepared cells from tissues, performed flow cytometry and F.J.M. analyzed the data with input from A.M.B. R.F.D. and W.B.M. synthesized the PET ligand. F.J.M., A.M.B., and P.S.S. carried out PET-imaging studies. Y.D. conducted the PET-imaging data analyses with input from and M.G.P.; F.J.M., and A.M.B., conducted statistical analyses of PET-imaging data. K.A.M.P., C.L., and B.S. designed and conducted necropsy with assistance from F.J.M. and A.M.B.; A.M.B. and M.C. conducted and analyzed immunochemistry.

Acknowledgements. A.M.B. acknowledges Gilbert Green for training received in the early stages of this project.

\section{Supplementary Materials}

Fig. 1S. No significant differences in viral load in chronically-HIV infected humanized mice at 10 and 16weeks post-infection. (A) ( $n=3,{\mathrm{HIV}-O P N^{+}}^{+}, n=8 \mathrm{HIV}-O P N^{-}$, one-way ANOVA). (B) Two-way ANOVA, $F=5.08$, $\mathrm{DFn}=1, \mathrm{Dfd}=18, P=.0369$.

Fig. 2S. Graphs with corresponding time-activity curves for early time periods, 8-10 minutes after ligand uptake for the indicated brain regions.

\section{References}


1. Boerwinkle A, Ances BM. Molecular Imaging of Neuroinflammation in HIV. Journal of neuroimmune pharmacology: the official journal of the Society on Neurolmmune Pharmacology. 2019;14(1):9-15.

2. Ghosn J, Taiwo B, Seedat S, Autran B, Katlama C. HIV. Lancet. 2018;392(10148):685-97.

3. Slim J, Saling CF. A Review of Management of Inflammation in the HIV Population. BioMed research international. 2016;2016:3420638.

4. Heaton RK, Franklin DR, Ellis RJ, McCutchan JA, Letendre SL, LeBlanc S, et al. HIV-associated neurocognitive disorder before and during the era of combination antiretroviral therapy: differences in rates, nature and predictors. J Neurovirol. 2011;17:3-16.

5. Heaton RK, Clifford DB, Franklin DR Jr, Woods SP, Ake C, Vaida F, et al. HIV-associated neurocognitive disorders persist in the era of potent antiretroviral therapy: CHARTER Study. Neurology. 2010;75:2087-96.

6. Maki PM, Rubin LH, Springer G, Seaberg EC, Sacktor N, Miller EN, et al Differences in Cognitive Function Between Women and Men With HIV. Journal of Acquired Immune Deficiency Syndromes (1999). 2018;79(1):101-7.

7. McArthur J, Steiner J, Sacktor N, Nath A. Human immunodeficiency virus-associated neurocognitive disorders: Mind the gap. Ann Neurol. 2010;67:699-714.

8. Jack CR Jr, Bennett DA, Blennow K, Carrillo MC, Dunn B, Haeberlein SB, et al. NIA-AA Research Framework: Toward a biological definition of Alzheimer's disease. Alzheimer's dementia: the Journal of the Alzheimer's Association. 2018;14(4):535-62.

9. Saylor D, Dickens AM, Sacktor N, Haughey N, Slusher B, Pletnikov M, et al. HIV-associated neurocognitive disorder - pathogenesis and prospects for treatment. Nat Rev Neurol. 2016;12:23448.

10. Kaul M. HIV's double strike at the brain: neuronal toxicity and compromised neurogenesis. Front Biosci. 2008;13:2484-94.

11. Prinz M, Jung S, Priller J. Microglia Biology: One Century of Evolving Concepts. Cell. 2019;179(2):292-311.

12. Perdiguero EG, Geissmann F. The development and maintenance of resident macrophages. Nat Immunol. 2016;17(1):2-8.

13. Salter MW, Stevens B. Microglia emerge as central players in brain disease. Nat Med. 2017;23(9):1018-27.

14. Bennett ML, Bennett FC, Liddelow SA, Ajami B, Zamanian JL, Fernhoff NB, et al. New tools for studying microglia in the mouse and human CNS. Proc Natl Acad Sci USA. 2016;113(12):E1738-46.

15. Boska MD, Dash PK, Knibbe J, Epstein AA, Akhter SP, Fields N, et al. Associations between brain microstructures, metabolites, and cognitive deficits during chronic HIV-1 infection of humanized mice. Mol Neurodegener. 2014;9:58.

16. Dash P, Gorantla S, Gendelman H, Knibbe J, Casale G, Makarov E, et al. Loss of neuronal integrity during progressive HIV-1 infection of humanized mice. J Neurosci. 2011;31:3148-57. 
17. Gorantla S, Makarov E, Finke-Dwyer J, Castanedo A, Holguin A, Gebhart C, et al. Links between progressive HIV-1 infection of humanized mice and viral neuropathogenesis. Am J Pathol. 2010;177:2938-49.

18. Guilarte TR. TSPO in diverse CNS pathologies and psychiatric disease: A critical review and a way forward. Pharmacol Ther. 2019;194:44-58.

19. Silva K, Hope-Lucas C, White T, Hairston T, Rameau T, Brown A. Cortical neurons are a prominent source of the proinflammatory cytokine osteopontin in HIV-associated neurocognitive disorders. J Neurovirol. 2015;21:174-85.

20. Burdo TH, Ellis RJ, Fox HS. Osteopontin is increased in HIV-associated dementia. J Infect Dis. 2008;198:715-22.

21. Marcondes MCG, Lanigan CMS, Burdo TH, Watry DD, Fox HS. Increased expression of monocyte CD44v6 correlates with the development of encephalitis in rhesus macaques infected with simian immunodeficiency virus. J Infect Dis. 2008;197:1567-76.

22. Brown A, Islam T, Adams R, Nerle S, Kamara M, Eger C, et al. Osteopontin enhances HIV replication and is increased in the brain and cerebrospinal fluid of HIV-infected individuals. J Neurovirol. 2011;17:382-92.

23. Calvez M, Hseeh G, Benzer S, Brown AM. Osteopontin counters human immunodeficiency virus type 1 -induced impairment of neurite growth through mammalian target of rapamycin and beta-integrin signaling pathways. Journal of Neurovirology. 2019;25(3):384-96.

24. Brown A. Osteopontin. A key link between immunity, inflammation and the central nervous system. Translational Neuroscience. 2012;3:288-93.

25. Cheng-Mayer C, Weiss C, Seto D, Levy JA. Isolates of human immunodeficiency virus type 1 from the brain may constitute a special group of the AIDS virus. Proc Natl Acad Sci USA. 1989;86:8575-9.

26. Mi Z, Guo H, Russell MB, Liu Y, Sullenger BA, Kuo PC. RNA aptamer blockade of osteopontin inhibits growth and metastasis of MDA-MB231 breast cancer cells. Mol Ther. 2009;17(1):153-61.

27. Thominiaux C, Dolle F, James ML, Bramoulle Y, Boutin H, Besret L, et al. Improved synthesis of the peripheral benzodiazepine receptor ligand [11C]DPA-713 using [11C]methyl triflate. Applied radiation and isotopes: including data, instrumentation and methods for use in agriculture. industry medicine. 2006;64(5):570-3.

28. Rubin LH, Sacktor N, Creighton J, Du Y, Endres CJ, Pomper MG, et al. Microglial activation is inversely associated with cognition in individuals living with HIV on effective antiretroviral therapy. AIDS. 2018;32(12):1661-7.

29. Coughlin JM, Wang Y, Ma S, Yue C, Kim PK, Adams AV, et al. Regional brain distribution of translocator protein using [(11)C]DPA-713 PET in individuals infected with HIV. J Neurovirol. 2014;20(3):219-32.

30. Boutin H, Chauveau F, Thominiaux C, Kuhnast B, Gregoire MC, Jan S, et al. In vivo imaging of brain lesions with [(11)C]CLINME, a new PET radioligand of peripheral benzodiazepine receptors. Glia. 2007;55(14):1459-68. 
31. Cosenza-Nashat M, Zhao ML, Suh HS, Morgan J, Natividad R, Morgello S, et al. Expression of the translocator protein of $18 \mathrm{kDa}$ by microglia, macrophages and astrocytes based on immunohistochemical localization in abnormal human brain. Neuropathol Appl Neurobiol. 2009;35(3):306-28.

32. Banati RB, Middleton RJ, Chan R, Hatty CR, Kam WW, Quin C, et al. Positron emission tomography and functional characterization of a complete PBR/TSPO knockout. Nature communications. 2014;5:5452.

33. Betlazar C, Harrison-Brown M, Middleton RJ, Banati RB, Liu G-J. Cellular sources and regional variations in the expression of the neuroinflammatory marker translocator protein (TSPO) in the normal brain. Inter J Mol Sci. 2018;19:2707.

34. Nutma E, Stephenson J, Gorter R, de Bruin J, Boucherie D, Donat C, et al. A quantitative neuropathological assessment of translocator protein expression in multiple sclerosis. Brain. 2019;142:3440-55.

35. Davalos D, Grutzendler J, Yang G, Kim JV, Zuo Y, Jung S, et al. ATP mediates rapid microglial response to local brain injury in vivo. Nat Neurosci. 2005;8(6):752-8.

36. Nimmerjahn A, Kirchhoff F, Helmchen F. Resting microglial cells are highly dynamic surveillants of brain parenchyma in vivo. Science. 2005;308(5726):1314-8.

37. Bennett ML, Bennett FC. The influence of environment and origin on brain resident macrophages and implications for therapy. Nature neuroscience. 2020;23(2):157-66.

38. Butovsky O, Jedrychowski MP, Cialic R, Krasemann S, Murugaiyan G, Fanek Z, et al. Targeting miR155 restores abnormal microglia and attenuates disease in SOD1 mice. Ann Neurol. 2015;77(1):7599.

39. Buttgereit A, Lelios I, Yu X, Vrohlings M, Krakoski NR, Gautier EL, et al. Sall1 is a transcriptional regulator defining microglia identity and function. Nature immunology. 2016;17(12):1397-406.

40. Hammond TR, Dufort C, Dissing-Olesen L, Giera S, Young A, Wysoker A, et al. Single-Cell RNA Sequencing of Microglia throughout the Mouse Lifespan and in the Injured Brain Reveals Complex Cell-State Changes. Immunity. 2019;50(1):253 - 71.e6.

41. Ladwig A, Rogall R, Hucklenbroich J, Willuweit A, Schoeneck M, Langen KJ, et al. Osteopontin Attenuates Secondary Neurodegeneration in the Thalamus after Experimental Stroke. Journal of neuroimmune pharmacology: the official journal of the Society on Neurolmmune Pharmacology. 2019;14(2):295-311.

42. Liaw L, Birk DE, Ballas CB, Whitsitt JS, Davidson JM, Hogan BL. Altered wound healing in mice lacking a functional osteopontin gene (SPP1). J Clin Invest. 1998;101:1468-78.

43. Rittling SR, Matsumoto H, McKee M, Nanci A, An X-R, Novick K, et al. Mice lacking osteopontin show normal development and bone structure but display altered osteoclast formation in vitro. $\mathrm{J}$ Bone Miner Res. 1998;13:1101-11.

44. Mankowski JL, Queen SE, Tarwater PJ, Adams RJ, Guilarte TR. Elevated peripheral benzodiazepine receptor expression in simian immunodeficiency virus encephalitis. Journal of Neurovirology. 
2003;9(1):94-100.

45. Hammoud DA, Endres CJ, Hammond E, Uzuner O, Brown A, Nath A, et al. Imaging serotonergic transmission with [11C]DASB-PET in depressed and non-depressed patients infected with HIV. Neurolmage. 2010;49(3):2588-95.

46. Garvey LJ, Pavese N, Politis M, Ramlackhansingh A, Brooks DJ, Taylor-Robinson SD, et al. Increased microglia activation in neurologically asymptomatic HIV-infected patients receiving effective ART. AIDS. 2014;28(1):67-72.

47. Vera JH, Ridha B, Gilleece Y, Amlani A, Thorburn P, Dizdarevic S. PET brain imaging in HIV-associated neurocognitive disorders (HAND) in the era of combination antiretroviral therapy. Eur J Nucl Med Mol Imaging. 2017;44(5):895-902.

48. Hammoud DA, Endres CJ, Chander AR, Guilarte TR, Wong DF, Sacktor NC, et al. Imaging glial cell activation with [11C]-R-PK11195 in patients with AIDS. Journal of Neurovirology. 2005;11(4):346-55.

49. Welikovitch LA, Do Carmo S, Magloczky Z, Malcolm JC, Loke J, Klein WL, et al. Early intraneuronal amyloid triggers neuron-derived inflammatory signaling in APP transgenic rats and human brain. Proceedings of the National Academy of Sciences of the United States of America. 2020.

50. Everall I, Vaida F, Khanlou N, Lazzaretto D, Achim C, Letendre S, et al. Cliniconeuropathologic correlates of human immunodeficiency virus in the era of antiretroviral therapy. J Neurovirol. 2009;15:360-70.

51. Tavazzi E, Morrison D, Sullivan P, Morgello S, Fischer T. Brain inflammation is a common feature of HIV-infected patients without HIV encephalitis or productive brain infection. Curr HIV Res. 2014;12:97-110.

52. Chang L, Shukla DK. Imaging studies of the HIV-infected brain. Handbook of Clinical Neurology. 2018;152:229-64.

53. Spudich S, Robertson KR, Bosch RJ, Gandhi RT, Cyktor JC, Mar H, et al. Persistent HIV-infected cells in cerebrospinal fluid are associated with poorer neurocognitive performance. J Clin Invest. 2019;129(8):3339-46.

54. Denhardt DT, Guo X. Osteopontin. A protein with diverse functions. FASEB J. 1993;7:1475-82.

55. Mark MP, Prince CW, Gay S, Austin RL, Butler WT. 44-kDal bone phosphoprotein (osteopontin) antigenicity at ectopic sites in newborn rats: kidney and nervous tissues. Cell Tissue Res. 1988;251:23-30.

\section{Figures}



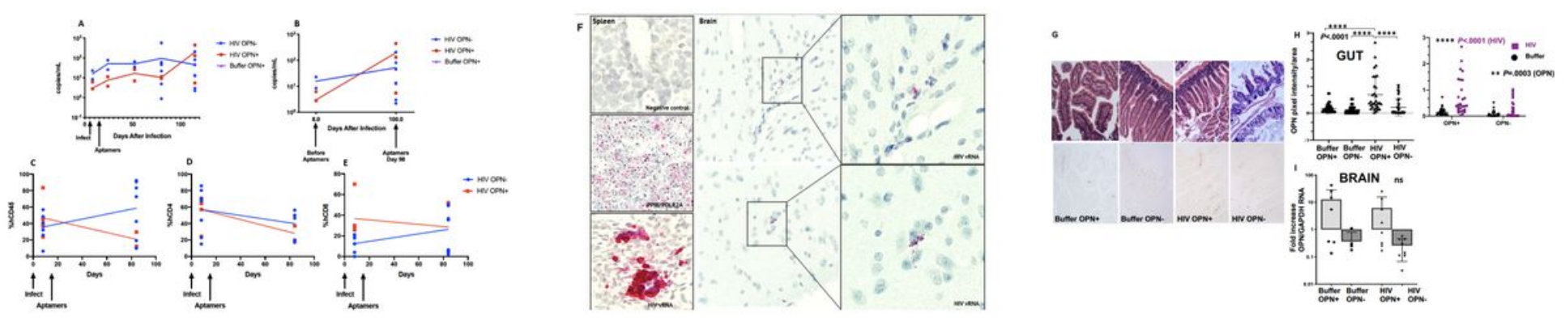

\section{Figure 1}

Viral load and immune cell subsets in chronically infected HIVSF162 humanized mice suppressed or not for osteopontin gene and protein expression. (A) Viral loads measured by Taqman qRT-PCR assay with a sensitivity of detection of $1 \mathrm{copy} / \mathrm{ml}$, varied among individual female mice (symbols) (blue, HIV-OPN-, $\mathrm{n}=8$; red, HIV-OPN+, $\mathrm{n}=3$ ) in each group (simple linear regressions, no significance differences in slope, $\mathrm{F}=$ 1.471. $D F n=2, D F d=44, P=0.2408$ ). (B) Similarly, no significant differences in viral load between the HIV OPN+ and HIV OPN- groups before or after aptamer treatment were found $(F=1.329 . D F n=2, D F d=13$, $P=0.2983$ ). (C-E) Percentage of white blood cells in plasma of HIV OPN+ (red) and HIV OPN- (blue) female mice before HIV infection and 85 days after aptamer treatment; (C) Absolute percentage of human CD45+ cells (HIV OPN+, red, $n=5$, start; $n=2$ end; HIV OPN- (blue, $n=7$, start; $n=7$ end; $F=3.800 . D F n=1, D F d=17$, $P=0.0680$ ), (D) percentage of hCD4 in the total hCD3+ T-cell population (HIV OPN+, red, $n=5$, start; $n=2$ end; $F=0.3051$. $D F n=1, D F d=17, P=0.5879$ ); HIV OPN+ (blue, $n=8$, start; $n=6$ end) and ( $E$ ) percentage of hCD8+ in the total hCD3+ T-cell population (HIV OPN+, red, $n=5$, start; $n=2$ end; HIV OPN- (blue, $n=7$, start; $n=7$ end; $F=1.489$. $D F n=1$, $D F d=17, P=0.2390)$. $(F)$ RNAscope was used to detect HIV RNA in spleen and brain of HIV-infected mice. Panels on left (top to bottom): negative control for DapB, positive controls (green=PPIB DNA, red=POLR2A, RNA), HIV RNA in spleen. Middle panel: HIV RNA in brain parenchyma with enlarged panels on the right showing localization to cytoplasm of cells. (G) Representative sections of gut (small intestines) stained with hematoxylin/eosin (top panels) or immunostained for OPN protein (lower panels). (H) Quantification of OPN label from female mice subjected to neuroimaging $(n=3,9-12$ sections per mouse) intensity (upper left panel, one-way ANOVA, $P=.0001$ ); there was an interaction between HIV and OPN (two-way ANOVA, $F=6.56 . \mathrm{DFn}=1, \mathrm{DFd}=104, \mathrm{P}=0.0118$ ) and independently with HIV that accounted for $15.02 \%$ of the total variance (two-way ANOVA, $F=21.96$. DFn $=1, D F d=104, P<$ 0.0001 ) and with OPN that was responsible for $9.36 \%$ of the total variance (two-way ANOVA, $F=13.69$. $\mathrm{DFn}=1, \mathrm{DFd}=104, \mathrm{P}=0.0003$ ). 


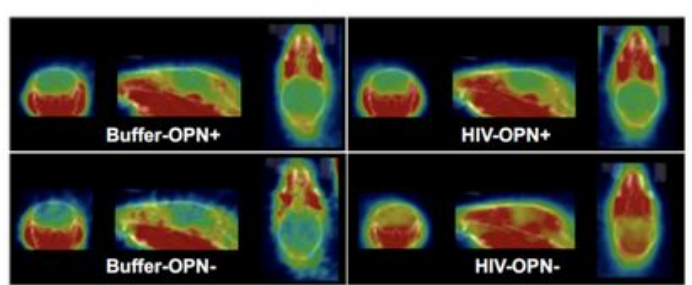

$\begin{array}{lll}0 & \% \mathrm{ID} / \mathrm{g} & 5\end{array}$
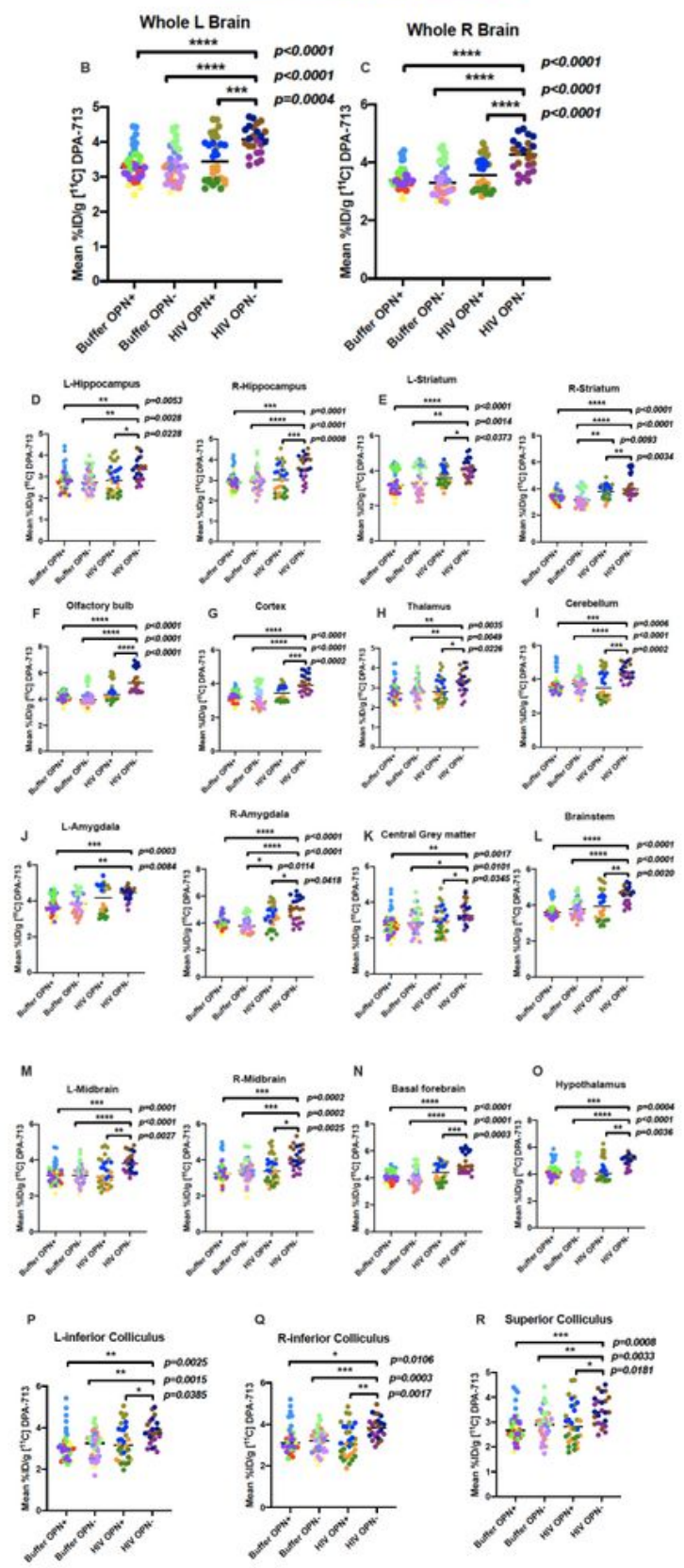

\section{Figure 2}

Increased uptake intensity of TSPO ligand DPA-713 in multiple brain regions in mice with global decreased expression of OPN. (A) Representative images after PET-CT reconstructed from a 45-60 min time period after injection of [11C] DPA-713 are shown. (B-O) Measurements were calculated over the 2050 min uptake time period in the brain regions indicated are shown ( $n=4$, Buffer-OPN+, $n=5$, Buffer-OPN-, 
$\mathrm{n}=4$, HIV-OPN+, n=3, HIV-OPN + ) with each mouse indicated with a different color, ordinary one-way ANOVA with Tukey's multiple comparisons, with mean and standard deviation, and significance shown).
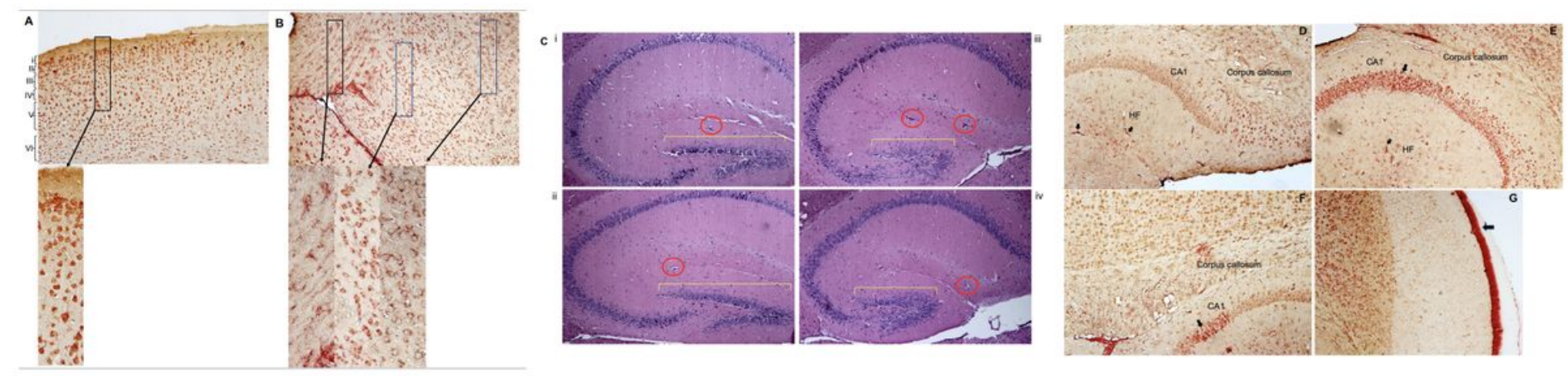

\section{Figure 3}

TSPO-Iba-1 double label immunoreactivity is abundant in cortical and hippocampal regions. (A-F) Representative samples of double-label immunostained $5 \square \mathrm{m}$ brain sections for TSPO (red) and lba-1 (brown). (A) Multiple layers (i-vi) of the cortex can be seen from buffer-OPN+ group. (B): Midbrain region from HIV-OPN+ group, showing a diversity of microglia morphologies. (C) HE stained hippocampal brain regions (i) Buffer-OPN+, (ii) Buffer-OPN-, (iii) HIV OPN+, (iv) HIV OPN-. Yellow bars, highlight the dentate gyrus and red circles indicate hippocampal fissures. (D-E) hippocampus of HIV-OPN-, (F) hippocampus in buffer-OPN- and (G) meningeal inflammation in HIV OPN+. 


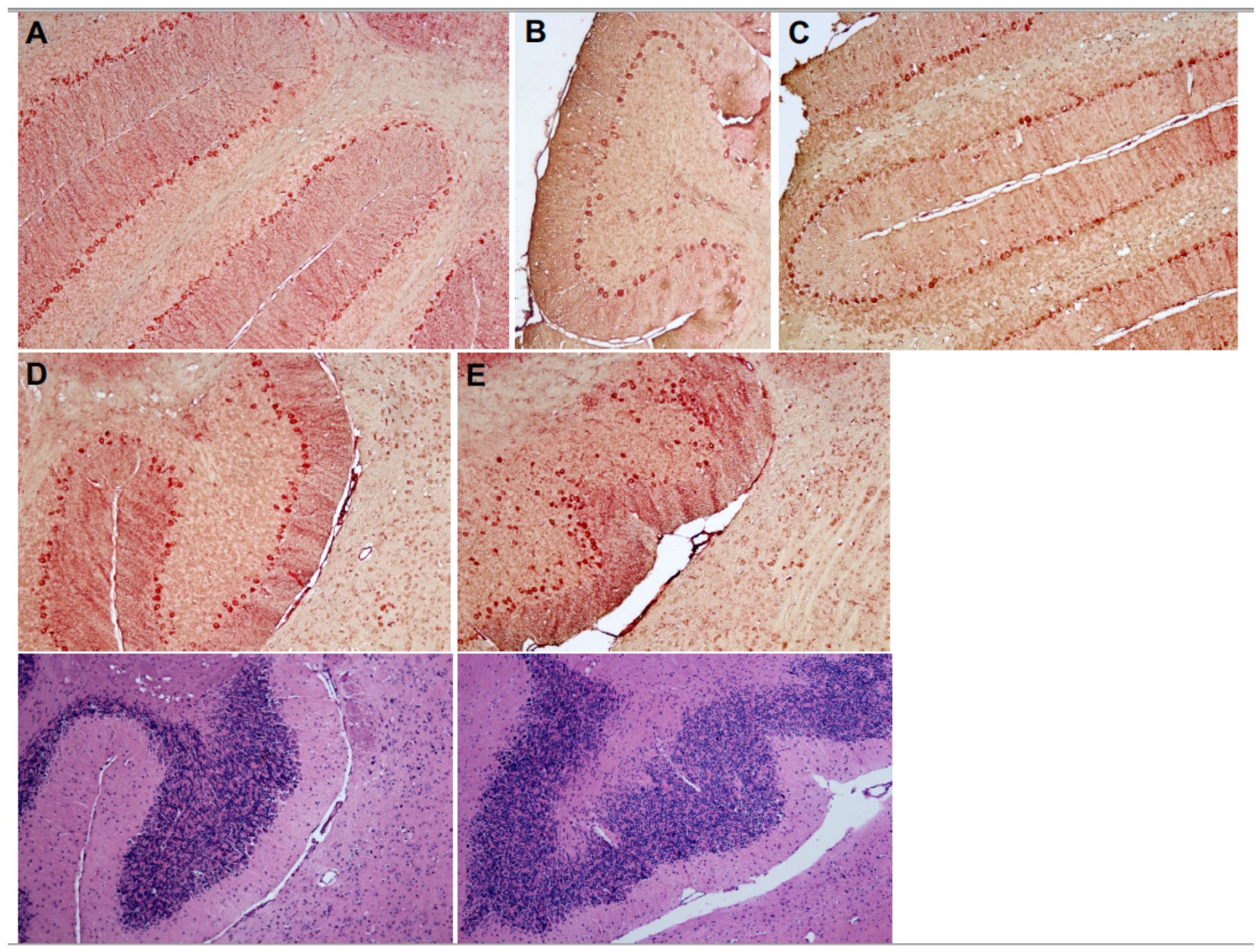

Figure 4

Robust TSPO immunoreactivity in a subset of neurons in the cerebellum. (A-E) Representative samples of double-label immunostained $5 \square \mathrm{m}$ brain sections for TSPO (red) and Iba-1 (brown) are shown (A) BufferOPN+, (B) HIV-OPN+), (C) HIV-OPN+, (D) Buffer-OPN-, (E) HIV-OPN-. The disordered array of Purkinje neurons in HIV-infected mice lacking osteopontin (E, HIV-OPN-) can be seen compared to (D, HIV-OPN+); the corresponding hematoxylin/eosin near adjacent section is shown. 


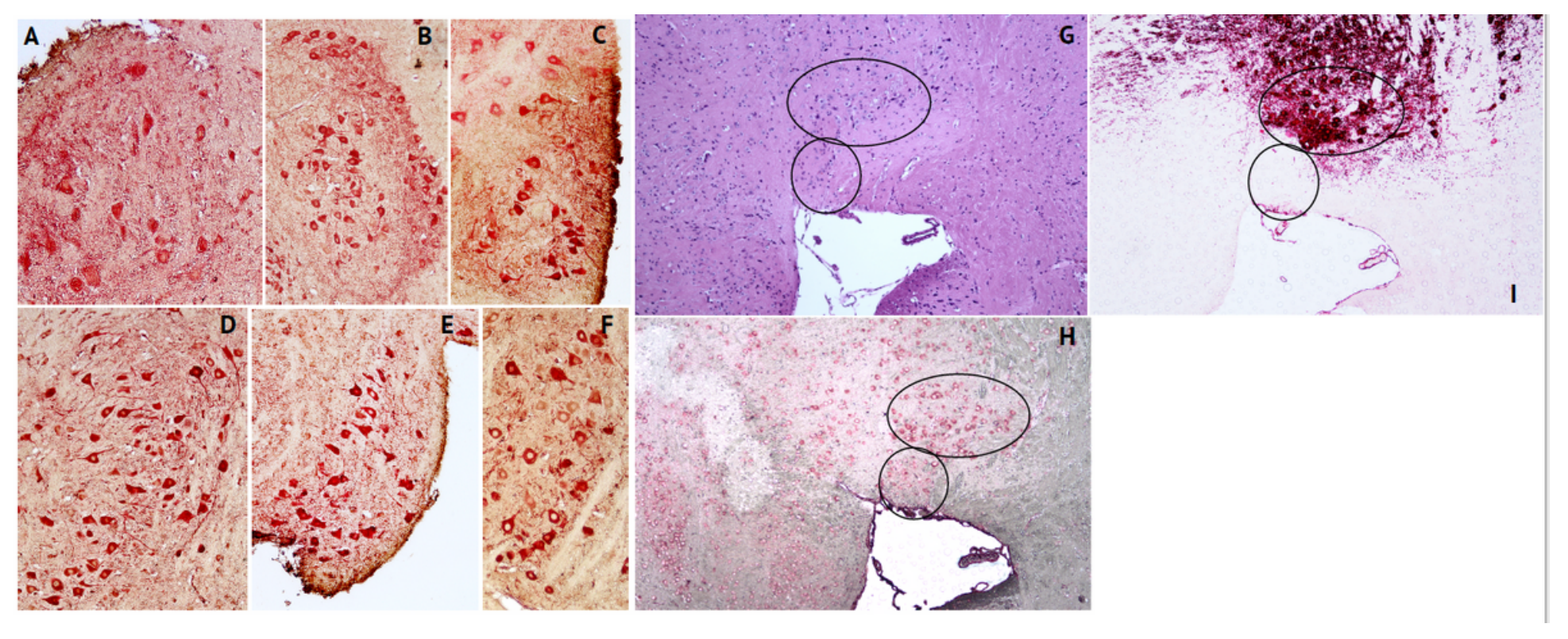

Figure 5

Strong TSPO immunoreactivity in a subset of tyrosine hydroxylase+ neurons in the striatum. (A-F) Representative samples of double-label immunostained $5 \square \mathrm{m}$ brain sections for TSPO (red) and Iba-1 (brown). (A) buffer OPN+, (B) HIV OPN+, (C) HIV OPN+, (D) Buffer OPN-, (E) HIV OPN-, (F) Buffer OPN-. (G) hematoxylin/eosin stained section, and serial adjacent sections stained with $(\mathrm{H})$ TSPO and (I) tyrosine hydroxylase. The black ovals demarcate the region of interest in each section. 
A

One-way ANOVA

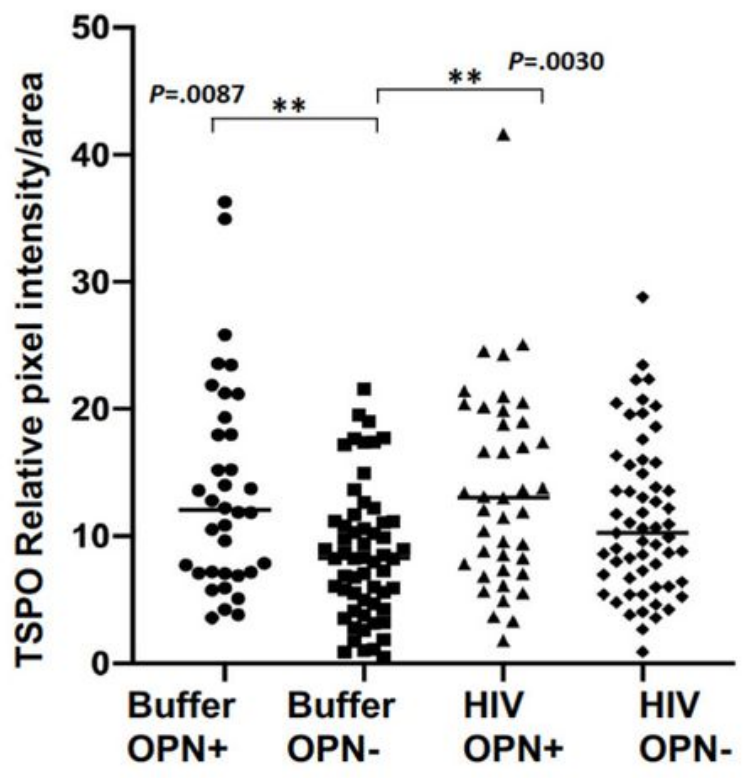

C

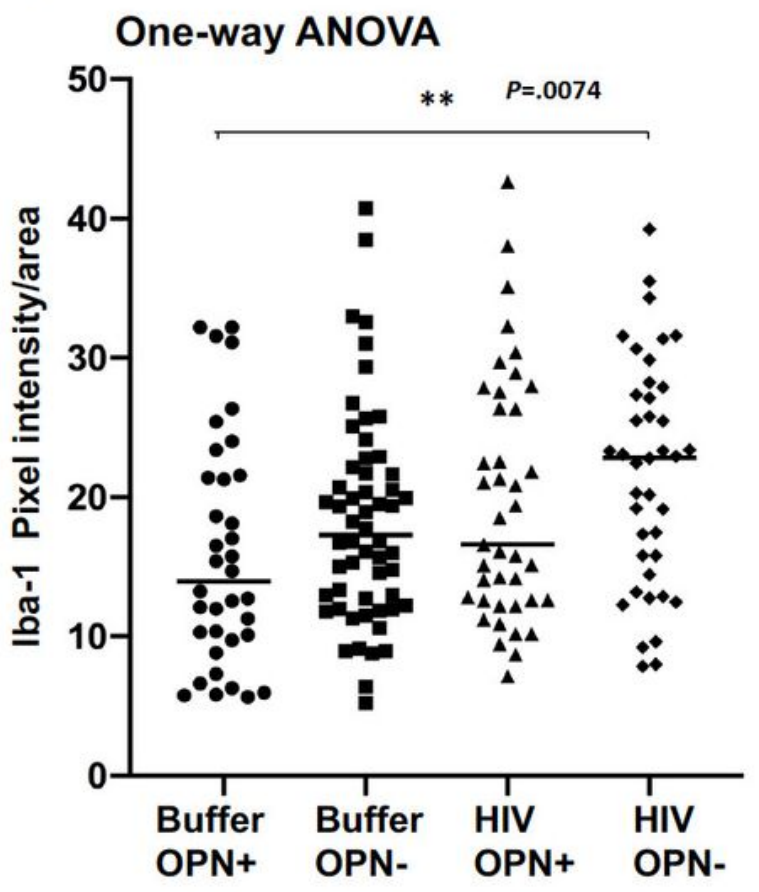

B

Two-way ANOVA

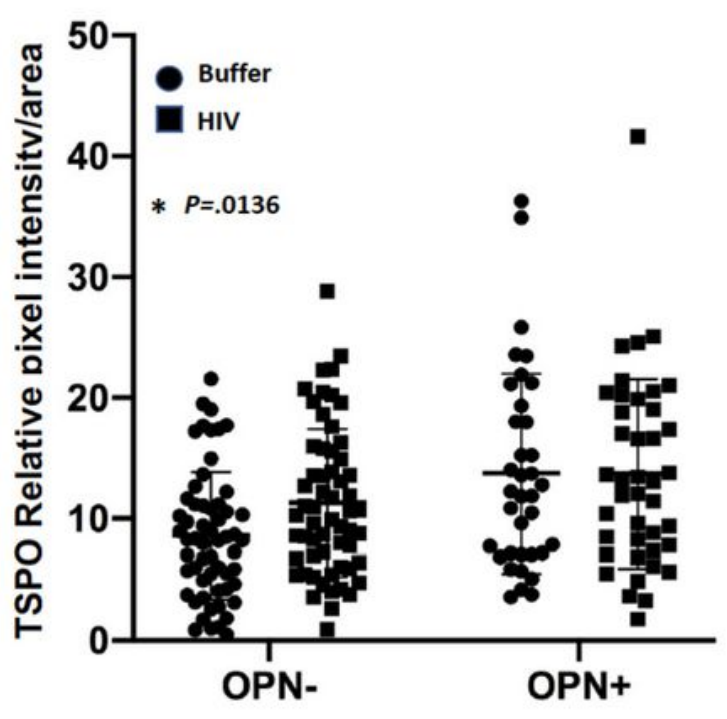

D

Two-way ANOVA

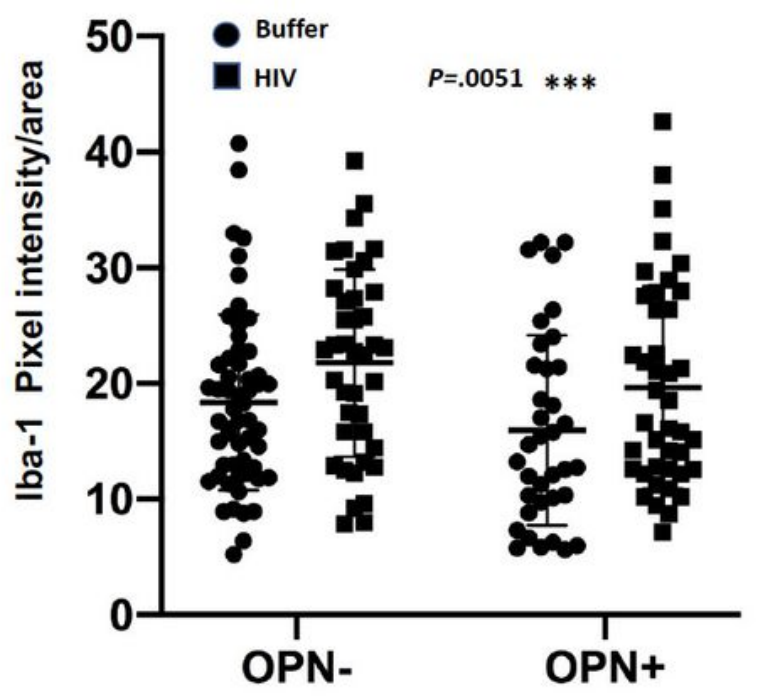

Figure 6

Iba-1, but not TSPO immunoreactivity is increased in the brains of mice suppressed for osteopontin. (A-D) Quantitative analyses of TSPO or Iba- 1 antibody reactivity, Buffer-OPN+ n=3; Buffer-OPN- n=5, HIV-OPN+, $n=3 ;$ HIV-OPN-, $n=3$; one-way ANOVA with Krusal-wallis test, Dunn's test or with two-way ANOVA to determine any interaction between osteopontin levels or HIV infection. The mean and standard deviation is shown. (A) While there was no difference in TSPO immunoreactivity in amongst the HIV groups (B) a 
significant interaction between TSPO levels and OPN ( $P=.0002)$ was observed. (D) A significant interaction between Iba-1 protein levels and HIV infection was found $(P=.0051)$.

\section{Supplementary Files}

This is a list of supplementary files associated with this preprint. Click to download.

- Fig1S.pdf

- FIGURE2SUPPLEMENTAL.pdf 